\title{
Belgeo
}

Revue belge de géographie

$1-2 \mid 2010$

Villes et grands équipements de transport/Cities and large transport infrastructures

\section{Villes et infrastructures de transport du Rhin supérieur : des grands équipements structurants?}

Cities and transport infrastructures in the Upper Rhine area: major structuring facilities?

Joël Forthoffer

\section{OpenEdition}

\section{Journals}

Édition électronique

URL : http://journals.openedition.org/belgeo/6972

DOI : $10.4000 /$ belgeo.6972

ISSN : 2294-9135

\section{Éditeur :}

National Committee of Geography of Belgium, Société Royale Belge de Géographie

\section{Édition imprimée}

Date de publication : 30 septembre 2010

Pagination : 147-158

ISSN : 1377-2368

\section{Référence électronique}

Joël Forthoffer, « Villes et infrastructures de transport du Rhin supérieur : des grands équipements structurants ? », Belgeo [En ligne], 1-2 | 2010, mis en ligne le 05 décembre 2012, consulté le 20 avril 2019. URL : http://journals.openedition.org/belgeo/6972 ; DOI : 10.4000/belgeo.6972

Ce document a été généré automatiquement le 20 avril 2019.

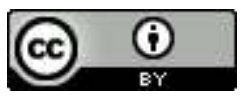

Belgeo est mis à disposition selon les termes de la licence Creative Commons Attribution 4.0 International. 


\section{Villes et infrastructures de transport du Rhin supérieur : des grands équipements structurants?}

Cities and transport infrastructures in the Upper Rhine area: major structuring facilities?

Joël Forthoffer

\section{Introduction}

1 La région du Rhin supérieur présente la singularité de réunir trois pays qui ont une volonté commune de développement. Les grands équipements et les projets correspondants, ferroviaires, aéroportuaires, routiers et plates formes plurimodales sont une des clefs du rayonnement futur de cette région qui ne peut se concevoir sans intégration dans les réseaux européens et mondiaux.

2 L'état des lieux des villes et des infrastructures de transport nous conduira à examiner, dans un second temps, l'effacement progressif de la frontière à l'appui d'exemples d'interactions de projets d'équipements communs mais également des disparités qui subsistent. 
Figure 1. Les agglomérations et villes du Rhin supérieur.

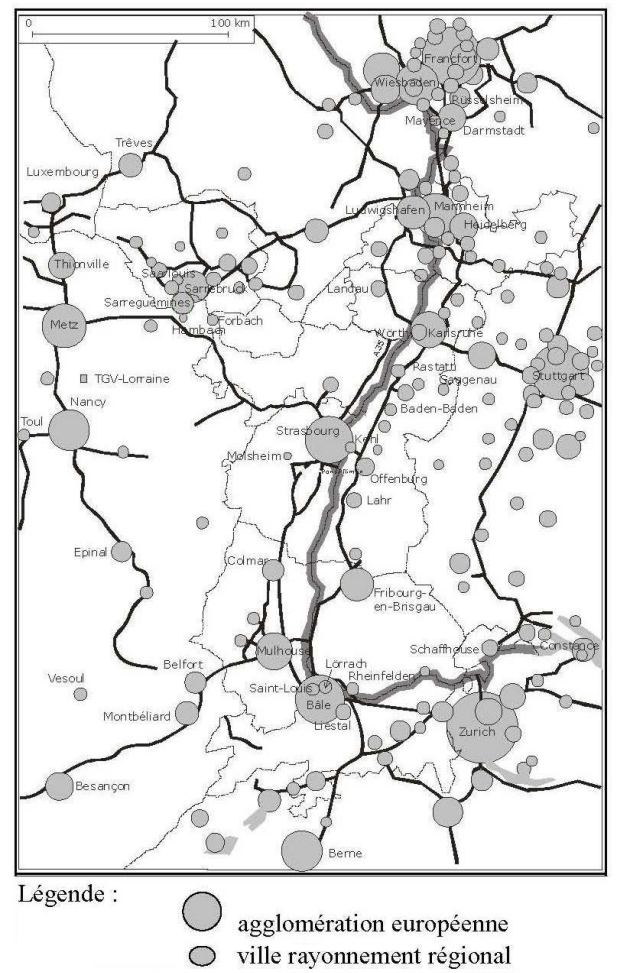

Source : WOESSNER (2007), L'Alsace territoire(s) en mouvement

\section{Les métropoles et les plates-formes d'échanges}

L'importance de sa superficie $\left(21000 \mathrm{~km}^{2}\right)$, la forte densité de son territoire (2 millions d'habitants), la pression foncière grandissante, l'utilisation croissante des espaces libres, l'urbanisation et la suburbanisation, l'augmentation du trafic de personnes et de marchandises, la mise en danger d'un écosystème sensible et la question de la mise en réseau transfrontalière des zones d'activités font que les défis d'un aménagement du territoire concerté au niveau du Rhin supérieur sont immenses.

Cet espace est caractérisé par une structure polycentrique assez unique en Europe. On n'y trouve pas une métropole unique, mais de nombreuses grandes villes (Bâle, Freiburg, Mulhouse, Strasbourg, Karlsruhe) et villes moyennes, dont la mise en réseau au niveau transfrontalier est très importante pour permettre d'exploiter efficacement cette particularité.

\section{Le maillage des villes, une résultante de l'histoire}

Dès les années 1960, Juillard (1968) évoquait les "cellules rhénanes", des régions de petites dimensions, mais riches de leurs villes comme de leurs campagnes peuplées et industrieuses.

Du fait de sa position centrale, au carrefour de plusieurs axes majeurs de communication européens, l'espace du Rhin supérieur est particulièrement concerné par le développement du secteur européen des transports et de la mobilité. Cela d'autant plus 
qu'il se trouve à la jonction de plusieurs politiques des transports pouvant se révéler contradictoires, voire concurrentes, et la structuration de l'espace métropolitain s'effectue encore trop selon les prérogatives nationales.

Tableau 1. Les réseaux urbains du Rhin supérieur.

\begin{tabular}{|c|c|c|}
\hline $\begin{array}{l}\text { Intitulé, } \\
\text { date de création, } \\
\text { habitants }\end{array}$ & $\begin{array}{l}\text { Ville- } \\
\text { centre }\end{array}$ & Fonctions supérieures remarquables \\
\hline $\begin{array}{l}\text { Rhein-Neckar, 2005, } \\
2,4 \text { millions }\end{array}$ & Mannheim & $\begin{array}{l}\text { - BASF, Heidelberger Druckmaschinen AG, ZDF. } \\
\text { - Technologie Park Heidelberg. } \\
\text { - Auto und Tecknik Museum Sinsheim, cathédrale de Spire } \\
\text { classée Patrimoine mondial UNESCO. }\end{array}$ \\
\hline $\begin{array}{l}\text { Trinationale } \\
\text { Agglomeration Basel } \\
\text { (ATB), } \\
2002 \\
0,6 \text { million }\end{array}$ & Bâle & $\begin{array}{l}\text { - Banque des Règlements Internationaux, UBS. } \\
\text { - Novartis, Roche, Ciba, Clariant, Actelion, Syngenta. } \\
\text { - EuroAirport, Panalpina, SBB Cargo. } \\
\text { - Internationaler Handball Federation. } \\
\text { - Baselword, Art'Basel, Fondation Beyeler, Kunstmuseum, } \\
\text { Scola Kantorum Basiliensis. }\end{array}$ \\
\hline $\begin{array}{l}\text { Eurodistrict } \\
\text { Strasbourg-Ortenau, } \\
2005 \text {, } \\
0,868 \text { million }\end{array}$ & Strasbourg & $\begin{array}{l}\text { - Conseil de l'Europe, Parlement européen, Cour Européenne } \\
\text { des Droits de l'Homme, Commission Centrale pour la } \\
\text { Navigation du Rhin, Eurocorps, Europol, Association des } \\
\text { Régions d'Europe. } \\
\text { - Ecole Nationale d'Administration. } \\
\text { - Arte, Burda Media, Europapark. } \\
\text { - Centre ville de Strasbourg classé Patrimoine mondial } \\
\text { UNESCO. }\end{array}$ \\
\hline
\end{tabular}

Source : FORTHOFFER (2009), Le déploiement du transport intermodal - L'espace rhénan et alpin

$7 \quad$ L'Allemagne joue la collégialité des villes depuis des dizaines d'années. La loi fédérale a créé 11 Metropolregionen, dont trois sont proches de l'Alsace. Francfort est la première place financière du pays; Stuttgart est davantage une technocité qui réfléchit aux voitures et aux camions de demain; Mannheim-Ludwigshafen se situe un ton plus bas, mais dispose avec Heidelberg de l'un des premiers pôles scientifiques européens. Ces trois "cellules rhénanes" développent des visions stratégiques grâce à leurs propres schémas d'aménagement.

En Suisse, avec les plans directeurs cantonaux, le système confédéral laisse l'initiative aux villes et cantons. La Metropolregion de Zurich est un peu moins peuplée que l'Alsace, mais elle brille des feux de la finance mondiale comme des prix Nobel de son école polytechnique. Bâle se définit elle-même comme "eine kleine Weltstadt", une petite villemonde, avec la pharmacie et les biotechnologies qui y connaissent un essor fulgurant. La coopération transfrontalière a permis de créer l'Agglomération Trinationale de Bâle (ATB). Son siège à Saint-Louis s'appuie sur un schéma d'urbanisme associant les communes des trois pays. 
9 En France, le jacobinisme a favorisé la concentration des fonctions nationales, puis mondiales, à Paris, ce qui freine l'émergence de Strasbourg. En 1949, Winston Churchill a propulsé Strasbourg dans le jeu européen. La ville peut ainsi rayonner grâce à sa fonction politique, et sans doute aussi grâce à sa culture. Quant à la région métropolitaine de Strasbourg, elle commence à exister depuis 2005 avec la création de l'Eurodistrict et des schémas de cohérence territoriale.

Imaginés par l'Etat français, les contrats métropolitains viennent à présent en renfort sans qu'il soit possible à ce jour de dire ce qu'ils valent vraiment. En signant un contrat métropolitain en 2007, Mulhouse se tourne vers le corridor Rhin-Rhône plutôt que vers Strasbourg; elle s'associe, en outre, à Colmar et à Fribourg avec un Eurodistrict encore balbutiant. Strasbourg n'est l'alliée de personne. Par conséquent, l'Alsace des villes n'existe pas.

11 Comme le souligne Woessner (2007), la structuration métropolitaine se fait selon "le génie propre à chacun".

\section{Les noeuds}

12 Préoccupés par la notion de centralité, les géographes ont émis de nombreuses théories. Les pionniers comme Thünen, Weber, Christaller et Lösch ont tenté de les modéliser. Ils ont lancé les bases fondamentales montrant les rapports interurbains, l'existence de liens permanents, l'importance des villes dans la distribution spatiale des flux humains et matériels ainsi que du façonnement de véritables hiérarchies urbaines. Christaller en 1933 (figure 2) s'appuie sur l'inégale importance des villes et leur répartition à l'intérieur de systèmes urbains fortement hiérarchisés. Il montre que les centres supérieurs donnent lieu à une hiérarchisation spécifique dont ressort une amorce de métropolisation. Mais il place à pied d'égalité Francfort et Zurich avec Strasbourg et Nuremberg, alors que Bâle, grande place financière n'est signalée qu'au second rang. 
Figure 2. L'Allemagne du Sud d'après Christaller.

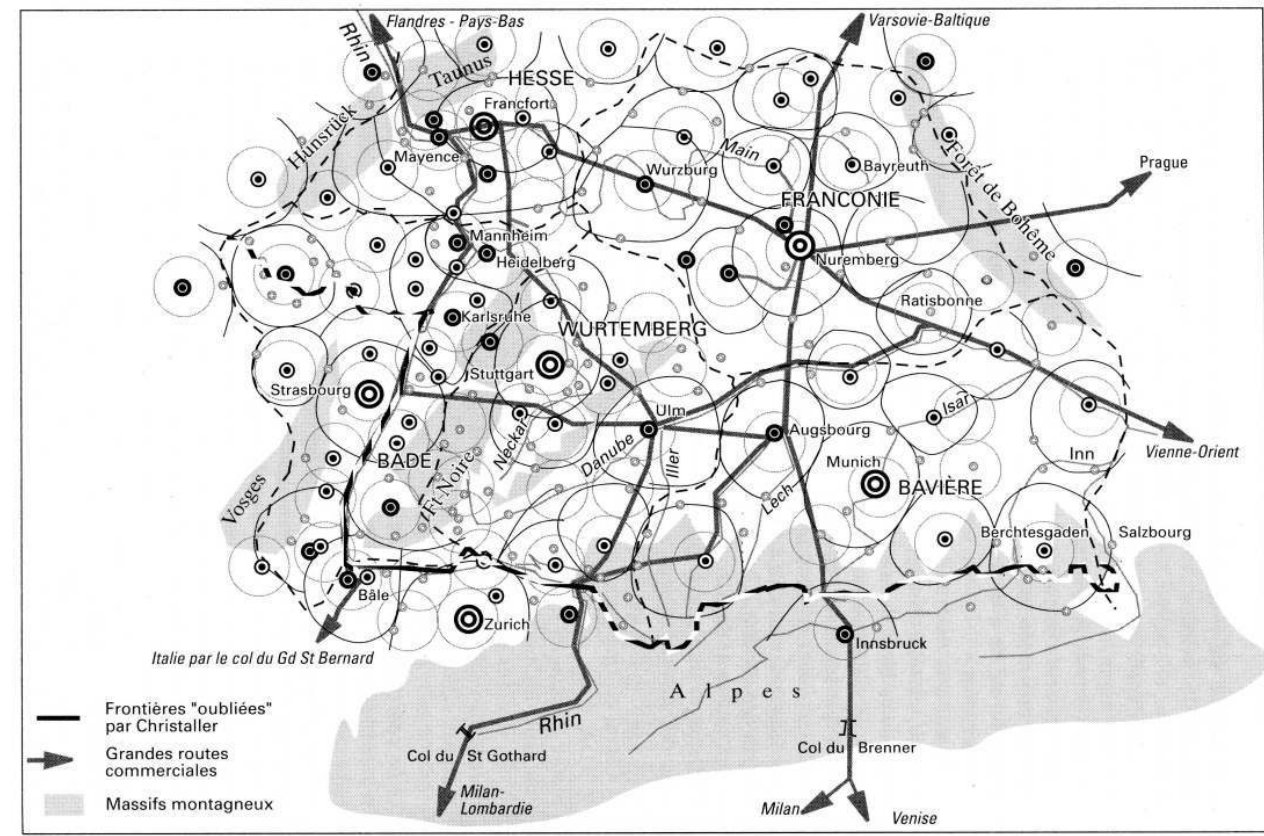

SOURCE : CHRISTALLER (1933), DIE ZENTRALEN ORTE IN SÜDDEUTSCHLAND

13 Jusqu'à la Seconde Guerre mondiale, la population se concentre dans les centres-villes et les zones contiguës pour y développer diverses activités industrielles, commerciales, de services,... A cette époque, les transports ne jouent qu'un rôle mineur compte tenu des faibles distances à parcourir et des moyens de transport qui font défaut. Seules les gares jouent un rôle de catalyseur de flux où convergeaient également les réseaux routiers. Elles deviennent de véritables pôles de développement au sein du tissu urbain, comme par exemple Bâle pour les voyageurs et les marchandises.

Aujourd'hui, les régions urbaines présentent un développement multipolaire qui n'exclut pas l'absence de structuration à la faveur des voies de communication, le tout se traduisant par la présence de pôles et d'axes de diffusion de l'habitat le long des routes conduisant vers ces pôles.

\section{Les flux majeurs}

Une étude, menée par la Direction régionale de l'équipement d'Alsace (2003) pour le compte de la conférence du Rhin supérieur, estime à plus de 4 millions par jour le nombre de déplacements de personnes à la fin du $\mathrm{XX}^{\mathrm{e}}$ siècle, hors déplacements intra-urbains et de courtes distances. Ces déplacements se répartissent pour $91 \%$ en interne, 8,5\% en échanges entre le Rhin supérieur et les autres régions européennes et en $0,5 \%$ de transit qui traverse le territoire sans s'y arrêter. Parmi les déplacements internes, le trafic transfrontalier représente une part de $5 \%$, soit près de 200000 déplacements quotidiens. Pour le transport de marchandises, l'estimation du trafic en 2002 s'élève à 365 millions de tonnes transportées en 1998 et 105 milliards de tonnes-kilomètres. Le trafic interne et d'échange atteint 290 millions de tonnes, soit 2,5 fois plus par habitant que l'Ile-deFrance! 

déficit et les lacunes (maillons manquants) des transports en commun à l'échelle de la région transfrontalière. Des éclairages complémentaires sont apportés aux études préalables en matière de transport en commun et d'inscription des réseaux dans les axes de communication majeurs régionaux et internationaux. Elle conclut et propose d'améliorer les infrastructures de communication et la desserte en transports en commun, avant tout dans leurs aspects transfrontaliers et de développer des liaisons entre les deux rives du Rhin.

\section{L'accessibilité urbaine et régionale}

17 En termes d'accessibilité, les principaux atouts du Rhin supérieur sont la diversité des moyens de transport, ferroviaire, aérien, routier et urbain, leur complémentarité à l'échelle du territoire et l'amélioration constante de l'offre transfrontalière et des interconnexions entre ces réseaux à l'échelle du territoire, et les réseaux de transport européens. Les principales caractéristiques de ces différents réseaux sont les suivantes :

- la région bénéficie de transports ferroviaires très récemment créés, modernisés et adaptés à la mobilité internationale : TGV Est Européen, trains régionaux transfrontaliers, connexions avec les réseaux allemands (ICE, DB Regio) et suisses, intermodalité avec les transports locaux :

- les transports aériens, routiers et fluviaux connaissent des mutations ou des revalorisations qui permettent de nouvelles utilisations ;

- les transports locaux des villes du territoire permettent la connexion des réseaux de transports internationaux aux équipements urbains.

Le tableau 2 présente les caractéristiques des différents modes de transport, les aspects transfrontaliers et leur projet de développement.

Tableau 2. Accessibilité de la région du Rhin supérieur.

\begin{tabular}{|l|l|l|l|}
\hline $\begin{array}{l}\text { Mode de } \\
\text { transport }\end{array}$ & Caractéristiques de \\
\hline $\begin{array}{l}\text { Train } \\
\text { grande } \\
\text { vitesse }\end{array}$ & $\begin{array}{l}\text { Arrivée du TGV Est Européen en juin } \\
\begin{array}{l}\text { Strasbourg devient le carrefour } \\
\text { européen des lignes à grande vitesse. }\end{array}\end{array}$ & $\begin{array}{l}\text { Aspect } \\
\text { transfrontalier }\end{array}$ & $\begin{array}{l}\text { Projet } \\
\text { développement }\end{array}$ \\
\hline $\begin{array}{l}\text { Trains } \\
\text { régionaux }\end{array}$ & $\begin{array}{l}\text { TER Alsace - DB Regio - CFF trafic } \\
\text { régional - OSB Rhône en } \\
\text { Déplacements transfrontaliers au } \\
\text { quotidien et connexion au réseau grande } \\
\text { vitesse ICE et TGV }\end{array}$ & $\begin{array}{l}\text { Magistrale pour } \\
\text { l'Europe jusqu'à } \\
\text { Budapest }\end{array}$ \\
\hline
\end{tabular}




\begin{tabular}{|c|c|c|c|}
\hline Avion & $\begin{array}{l}5 \text { aéroports sur le territoire : } \\
\text { l'EuroAirport Basel-Mulhouse-Freiburg, } \\
\text { l'aéroport international de Strasbourg- } \\
\text { Entzheim ; le Baden Airpark Söllingen; } \\
\text { l'aéroport de Lahr ; l'aéroport de Colmar } \\
\text { Houssen } \\
\text { L'aéroport international de Francfort }\end{array}$ & ** & $\begin{array}{l}\text { Développement des } \\
\text { compagnies low-cost } \\
\text { tram-train } \\
\text { permettant de relier } \\
\text { les aéroports aux } \\
\text { centres villes. }\end{array}$ \\
\hline Voie d'eau & $\begin{array}{l}11 \text { ports sur l'espace transfrontalier avec } \\
\text { une fonction importante de plaque } \\
\text { tournante: Strasbourg, Ottmarsheim, } \\
\text { Weil, Wörth, Karlsruhe, Germersheim et } \\
\text { Gambsheim } \\
\text { Croisières sur le Rhin }\end{array}$ & $* * *$ & $\begin{array}{l}\text { Lignes fluviale } \\
\text { conteneur } \\
\text { Port transfrontalier }\end{array}$ \\
\hline Route & $\begin{array}{l}\text { Nœud important du réseau routier et } \\
\text { autoroutier européen (HAFRABA) } \\
\text { Développement de solutions alternatives } \\
\text { dans l'utilisation de la route avec } \\
\text { covoiturage }\end{array}$ & ** & $\begin{array}{l}\text { Grand contournement } \\
\text { ouest de Strasbourg. } \\
3^{\text {ème }} \text { voie autoroute } \\
\text { Freiburg }\end{array}$ \\
\hline $\begin{array}{l}\text { Tramway/ } \\
\text { bus }\end{array}$ & $\begin{array}{l}\text { Extension des lignes urbaines: Bâle, } \\
\text { Mulhouse, Strasbourg }\end{array}$ & $* *$ & $\begin{array}{l}\text { Interconnexion avec } \\
\text { le train } \\
\text { Intermodalité } \\
\text { voiture-tram }\end{array}$ \\
\hline Vélo & $\begin{array}{l}\text { Réseau d'aménagements cyclables } \\
\text { Multiplication des parcs à vélos }\end{array}$ & *** & $\begin{array}{l}\text { Intermodalité entre } \\
\text { tram ou train et vélo }\end{array}$ \\
\hline
\end{tabular}

Source : FORTHOFFER (2003), Evolution des transports ferroviaires de marchandises en Europe besoins, revêt dès lors une importance stratégique toute particulière pour les années à venir, notamment en ce qui concerne la mise en réseau transfrontalière des systèmes de transport et l'élaboration de solutions spécifiques, apportant des réponses adaptées aux besoins et aux facteurs de difficultés propres à l'espace.

\section{Les agglomérations et le jeu transfrontalier}

21 La coopération transfrontalière est un instrument essentiel pour développer les territoires frontaliers situés généralement en périphérie des centres de décisions. De ce fait la notion même de frontière évolue bien qu'elle rencontre encore des lenteurs dans la programmation de ses infrastructures. 


\section{Le statut de la frontière}

Pour les agglomérations du Rhin supérieur, le statut même de la frontière peut être compris selon différentes approches (figure 3) :

a. Héritage de l'histoire, la frontière-barrière entre l'Alsace du Nord et le Rhénanie Palatinat est devenue une ligne poreuse. Elle se laisse traverser par de nombreux flux sans introduire de contrainte spécifique, bien que certains éléments diffèrent toujours selon les Etats (réglementations diverses, spécificités techniques).

b. De part et d'autre de la ligne, la frontière génère également un espace interface où l'on retrouve chez soi des éléments appartenant au pays voisin. Ainsi, l'Allemagne, et encore plus la Suisse, sont des pays de forte culture ferroviaire (cadencement des dessertes régionales...). Conception qui caractérise également l'Alsace avec les projets de tram-train de Mulhouse et Strasbourg.

c. La frontière forme une coupure entre des aires-systèmes propres à chaque pays et particulièrement difficiles à connecter. Les institutions restent d'abord nationales. Les entreprises agissent conformément à une culture nationale. Les réseaux de transport sont affectés par de nombreuses coupures ou difficultés comme l'interopérabilité ferroviaire : elle a conduit, en 2008 à abandonner la circulation du train régional suisse FLIRT (abréviation de l'allemand Flinker Leichter Innovativer Regional-Triebzug pour automotrice innovative agile et léger) Alsace en France.

d. Mais la question de la frontière mérite d'être dépassée : une région transfrontalière soumise à une intense territorialisation sera considérée comme étant au centre d'un nouveau système, et non plus sur les marges de deux systèmes. Des processus originaux de structuration apparaissent alors, à l'image de l'EuroAirport Bâle-Mulhouse-Fribourg ou des trains régionaux transfrontaliers.

Schématiquement, la construction a été marquée par différentes étapes avec la Regio Basiliensis en 1963 (TriRhena depuis 1996), la Conférence du Rhin supérieur depuis 1975, les découpages Interreg, les accords de Karlsruhe en 1996, le Groupement Local de Coopération Transfrontalière (GLCT) Fessenheim-Hartheim et les eurodistricts en 2005 (Strasbourg) - 2007 (Bâle). 
Figure 3. Statuts conceptuels pour la frontière.

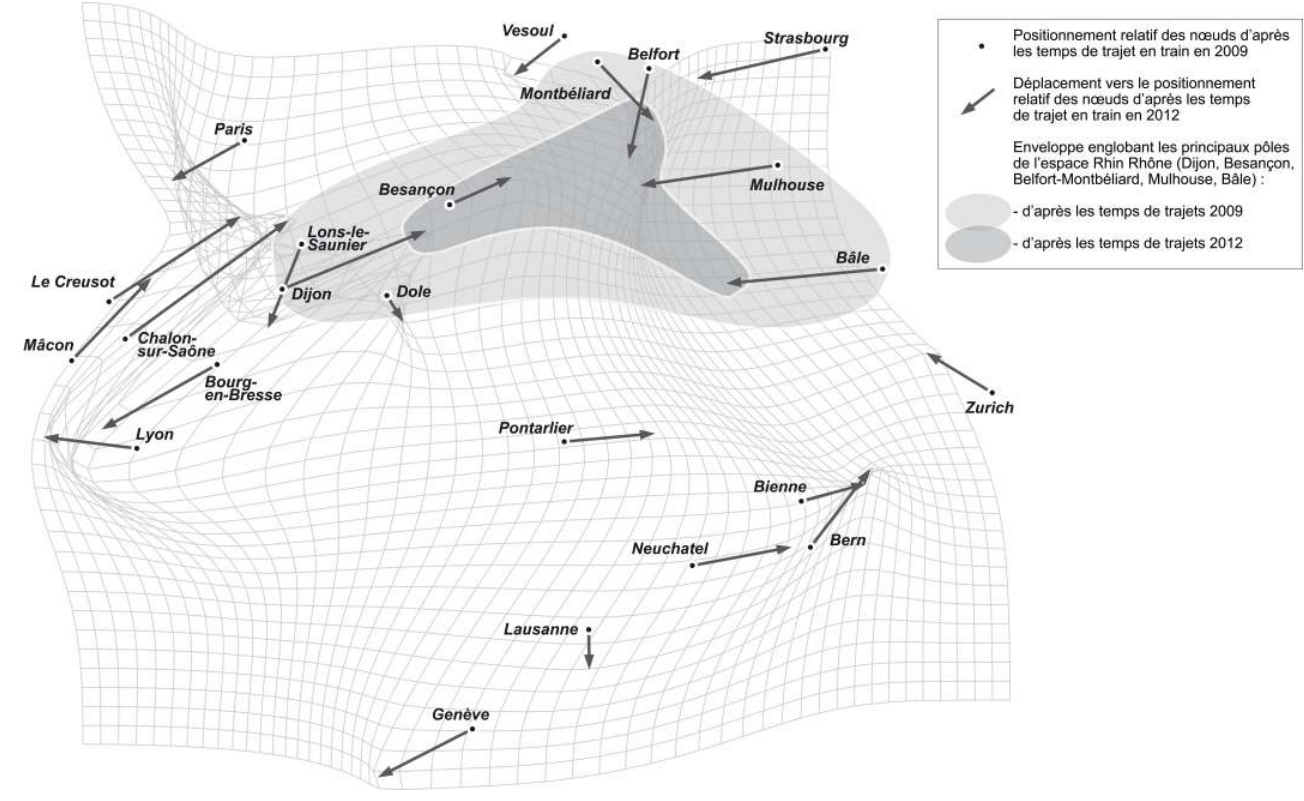

Source : Luis Felipe Menezes (2008), Mission Opérationnelle Transfrontalière

\section{Une volonté affichée de coopération}

\section{L'Eurodistrict Strasbourg-Ortenau}

Autrefois, Pierre Pflimlin, maire de Strasbourg de 1959 à 1983, avait souhaité la création d'un district fédéral européen; Emile Allais, prix Nobel d'économie, avait lui aussi fait cette suggestion dans les années 1990. En 2003, lors du $40^{\mathrm{e}}$ anniversaire du Traité de l'Elysée, MM. Chirac et Schröder désiraient "la création d'un Eurodistrict Strasbourg-Kehl, bien desservi, ayant vocation à explorer de nouvelles formes de coopération et à accueillir des institutions européennes".

Aujourd'hui, Strasbourg apparaît comme une métropole contrariée qui ne rayonne guère outre-Rhin. Pour l'Ortenau voisin, petite région placée à l'écart des grandes villes badoises (Karlsruhe et Fribourg-en-Brisgau), Strasbourg pourrait devenir une capitale régionale "naturelle", s'il n'y avait la coupure de la frontière nationale. Pourtant, l'Ortenau recèle des activités "structurantes" : les sièges d'entreprises (les éditions Burda, les robinets Grohe,...), le tourisme-loisirs avec l'Europa-Park classé au cinquième rang mondial, et les transports (le port de Kehl, la gare ICE d'Offenburg, l'aéroport de Lahr,...). Mais les complémentarités franco-allemandes semblent aussi évidentes que mal utilisées.

Le premier défi de l'eurodistrict consiste à articuler des entités institutionnelles aux compétences et aux modes de fonctionnement différents. Le périmètre du Schéma de Cohérence Territoriale de la Région de Strasbourg (SCOTERS) regroupe 581300 habitants et celui du Regionalplan de l'Ortenau 405607. Le syndicat mixte du SCOTERS et le Kreis d'Ortenau travaillent donc à leur mise en cohérence, même si le SCOTERS court de 2000 à 2015 et le Regionalplan de 1995 à 2010. Piloté par une assemblée élue au suffrage universel, le Kreis constitue une subdivision territoriale forte. 


\section{L'Agglomération Trinationale de Bâle (ATB)}

27 "Basel im Elsass" écrivait un chroniqueur du $\mathrm{XV}^{\mathrm{e}}$ siècle: voilà une représentation insoutenable aujourd'hui. Mais vue du ciel, l'agglomération bâloise, forte de 600000 habitants, constitue une seule et même entité et, de fait, s'étend sur trois pays, la Suisse, l'Allemagne et la France. La ville de Bâle est séparée du reste de la Suisse par la montagne du Jura alors que les vallées de la Forêt-Noire, la plaine rhénane et les collines du Sundgau lui offrent des possibilités d'extension en dehors de son territoire national. Au sein du système confédéral suisse, les Bâlois sont des techniciens rompus aux pratiques de la coopération avec d'autres entités quasiment souveraines dans de petits espaces. L'émergence d'une structure de coopération trinationale à l'échelle de l'agglomération semble donc possible et rationnelle.

Les partenaires des trois secteurs nationaux travaillent sur des études de faisabilité. Cette entreprise est possible parce que Bâle apparait comme une ville de projets. Elle dispose de deux observatoires de qualité, la Wirtschaftsstudie Nordwestschweiz (Etude économique du Nord-Ouest de la Suisse) dont les études sont largement débattues dans les cercles politiques, économiques et associatifs.

29 Malgré les difficultés juridiques, la ville de Bâle soutient les projets. Sa stratégie est le développement de l'agglomération. La ville constituait traditionnellement une plateforme financière, chimique et logistique. Sa politique d'urbanisme se traduit par l'accomplissement de projets successifs : la foire avec le Messeturm (l'immeuble le plus élevé de la Suisse), Euroville pour le quartier de la gare, le stade Saint-Jacques intégrant un centre commercial, les zones d'activités de la BioValley, bientôt un waterfront sur le Rhin, etc. Les communes françaises, allemandes et suisses cherchent à tirer avantage de la croissance bâloise dans un contexte de rareté foncière ; implicitement, elles tendent à s'aligner sur les normes bâloises en matière d'urbanisme. A Saint-Louis, par exemple, l'ancienne usine de spiritueux Fernet-Branca est devenue un centre d'art contemporain d'envergure internationale.

Bâle a réussi à développer un paradoxe : tout en conservant ses valeurs et ses élites traditionnelles, elle s'est ouverte à de nouveaux venus et aux comportements anglosaxons du monde de la science et de la finance. Les réseaux de l'ATB enjambent les régions proches au profit de relations avec d'autres métropoles du Rhin supérieur ou du monde, mais ils se déconnectent de leur voisine mulhousienne qui leur devient étrangère.

31 Récemment créés, ces eurodistricts, s'ils tentent de développer des projets d'équipements de transports comme des tramways transfrontaliers, n'arrivent pas encore à peser en tant qu'entité dans les grands projets d'équipements structurants du Rhin supérieur.

\section{Une réalité : encore des disparités dans les infrastructures}

Les réseaux d'infrastructures de transport présentent certes des lacunes, mais l'obstacle majeur demeure les franchissements du Rhin.

Du point de vue des infrastructures de transport routier, le Rhin supérieur se distingue par un réseau dense d'autoroutes, de routes nationales et régionales/départementales. Sa connexion aux centres économiques voisins, tels que Francfort, Paris, Stuttgart, Lyon, Bern, Zurich ou Milan est assurée. Le réseau routier du Rhin supérieur est caractérisé avant tout par des liaisons Nord-Sud très bien développées de part et d'autre du Rhin 
(Strasbourg-Mulhouse-Bâle d'un côté, Karlsruhe-Freiburg-Bâle de l'autre), qui relient entre eux les principaux centres économiques de la région. Il est néanmoins à mentionner que, du côté français la discontinuité autoroutière dans l'axe nord/sud forme au nord de Colmar un véritable goulet d'étranglement. A l'inverse, les liaisons Est-Ouest, qui se heurtent à la frontière naturelle du Rhin, sont moins développées. De plus il faut déplorer l'inadaptation du réseau au développement du trafic poids lourds, notamment dans les zones urbaines.

Le réseau ferroviaire de la région se caractérise également par une infrastructure remarquable, qui offre une très bonne connexion au réseau ferroviaire transeuropéen. Il s'articule toutefois lui aussi principalement autour des deux axes nord-sud nationaux (du côté français et du côté allemand), qui sont tous les deux très bien reliés au réseau ferroviaire suisse à hauteur de Bâle. La liaison Ouest-Est se concentre, quant à elle, sur le seul passage de la frontière existant, entre Strasbourg et Kehl. Le réseau alsacien est en rupture de capacité et ne possède pas encore de ligne à grande vitesse. Il faut également mentionner dans le transport ferroviaire interrégional l'importance grandissante pour le Rhin supérieur, des ponts ferroviaires Wörth-Karlsruhe et Germersheim, ainsi que du tunnel planifié de Rastatt.

Le Rhin supérieur compte cinq aéroports: l'aéroport de Bâle-Mulhouse (EuroAirport) avec 2,5 millions de passagers par an, celui de Strasbourg/Entzheim avec 2,1 millions de passagers, le Baden Airport Söllingen, qui connaît un essor impressionnant (20000 passagers en 1997 contre 711000 en 2005), l'aéroport de Lahr (réservé au trafic de fret à l'origine mais qui sera ouvert à l'avenir au trafic aérien de passagers, à destination notamment de l'Europapark à Rust), et enfin l'aéroport de Colmar-Houssen (aéroport à l'avenir incertain, ouvert au trafic de petits aéronefs).

Grâce au Rhin, la région du Rhin supérieur dispose également d'une liaison fluviale majeure avec la Mer du Nord, dont l'importance se mesure aux 37 millions de tonnes de marchandises qui y circulent chaque année. L'espace transfrontalier compte 11 ports avec une fonction importante de plaque tournante. Parmi ceux-ci, les ports de Bâle, Strasbourg, Ottmarsheim, Weil, Wörth, Karlsruhe, Germersheim et Gambsheim disposent des infrastructures nécessaires au transbordement de conteneurs.

Enfin se pose la question des ponts sur le Rhin. Le Rhin n'est plus une barrière, mais il demeure un obstacle pour trois raisons. La construction d'un pont coûte cher. Il s'agit de connecter deux systèmes avec deux cultures différentes de part et d'autre du fleuve. Les logiques évoluent: après une période routière, le rail revient; et les passerelles piétonnes-cyclistes constituent un nouvel élément emblématique de la coopération transfrontalière.

Les franchissements routiers ne connaissent qu'une liaison autoroutière, celle de l'A36 à Ottmarsheim (1976). A Strasbourg, le pont Pflimlin était inscrit au schéma directeur de 1973 et il a finalement été ouvert en octobre 2002. Il passe à deux voies en quittant la France ; sa portée est de 205 mètres pour une longueur totale de $957 \mathrm{~m}$. La France aurait souhaitée un raccordement autoroutier jusqu'à la HAFRABA ; l'Allemagne a préféré lui adjoindre des pistes cyclables...

39 Les franchissements ferroviaires apparaissent insuffisants. Seulement deux ponts ferroviaires à voie unique à Strasbourg et à Chalampé, connectent le trafic français à l'autre rive du Rhin. La situation contemporaine est moins favorable qu'en 1939: les 
ponts ferroviaires de Rastatt et de Huningue ont été détruits lors de la deuxième guerre mondiale, sans avoir été reconstruits.

Le sommet de La Rochelle (1992) avait acté l'idée de la reconstruction du pont ferroviaire à Strasbourg pour faciliter le passage du TGV; les travaux seront terminés en 2010. En 2009 , un autre pont est décidé pour la ligne $\mathrm{D}$ du tramway destinée à se rendre à la gare de Kehl. Chiffré à 42 millions d'euros, le démarrage des travaux est attendu en 2011.

41 A Mulhouse, les trains de fret traversent le Rhin par le pont à voie unique de Chalampé. Cette ligne est empruntée par les trains régionaux, aujourd'hui à titre expérimental le dimanche, et à partir de 2012 tous les jours. En outre les Fribourgeois attendent une desserte du TGV Rhin-Rhône dès décembre 2011.

Les passerelles piétonnes échappent à la convention franco-allemande de 1953 grâce aux accords de Karlsruhe de 1996. A l'échelle de l'agglomération de Strasbourg - Kehl, le Jardin des Deux Rives inauguré en 2004 transforme le no man's land du secteur du Pont de l'Europe en couture verte transfrontalière. La passerelle Mimram d'une portée de $183 \mathrm{~m}$ a coûté un peu plus de 11 millions d'euros. Dans l'agglomération de Bâle, la passerelle de Huningue - Weil-am-Rhein (2006) est, paraît-il, dotée de la portée la plus longue au monde (238 mètres) et a coûté 9 millions d'euros.

\section{Conclusion}

43 Après la première étape pionnière de partage des connaissances, et la deuxième étape de développement de liaisons transfrontalières, le Rhin supérieur entame une nouvelle étape avec la volonté de partager des projets communs d'aménagement d'infrastructures de transports. Le concept moderne de l'aménagement du territoire ne se limite plus à la question de l'harmonisation des planifications nationales et régionales, il se concentre bien plus sur l'élaboration et la mise en oeuvre d'actions transversales, en réseaux, dépassant les frontières et se donnant pour objectif un pilotage actif du développement d'ensemble de l'espace transfrontalier.

De plus, par son caractère transversal, la politique transfrontalière d'aménagement du territoire croise en de nombreux points les politiques sectorielles multiscalaires. Son défi principal lors des prochaines années sera justement de parvenir à faire évoluer l'orientation purement sectorielle de ces politiques, au profit d'une vision plus intégrée et axée autour des forces et faiblesses majeures de l'ensemble de l'espace du Rhin supérieur.

\section{BIBLIOGRAPHIE}

BAVOUX J.-J., CHARRIER J.-B. (1994), Transport et structuration de l'espace dans l'Union Européenne, Paris, Masson Géographie, $222 \mathrm{p}$.

BEAUJEU-GARNIER J. (1995), Géographie Urbaine, Paris, Armand Colin, U-Géographie, 349 p.

CHRISTALLER W. (1933), Die zentralen Orte in Süddeutschland, Iéna, Von Gustav Fischer, 100 p. 
DIRECTION RÉGIONALE EQUIPEMENT ALSACE (2003), Etude plurimodale des transports dans le Rhin Supérieur, Edition Conférence du Rhin Supérieur, 12 p.

FORTHOFFER J. (2003), “Evolution des transports ferroviaires de marchandises en Europe”, in AUPHAN E., DEZERT B. (éd.), L'Europe en mouvement - Population - Transports - Aménagements Tourisme, Paris, Ellipses, collection Transversales, pp. 51-61.

FORTHOFFER J. (2009), “Le déploiement du transport intermodal - L'espace rhénan et alpin”, L'identité européenne : ses institution et sa géopolitique, Paris, Société de Géographie, pp. 85-103.

JUILLARD J. (1968), L'Europe rhénane - Géographie d'un grand espace, Paris, Armand Colin, 292 p.

OUVRAGE COLLECTIF (2008), “Les territoires transfrontaliers : l'Europe au quotidien”, actes du colloque des 8 et 9 novembre 2007, Editions Missions Opérationnelle Transfrontalière (MOT), $182 \mathrm{p}$.

WOESSNER R. (2007), L'Alsace territoire(s) en mouvement, Colmar, Jérôme Do Bentzinger Editeur, $183 \mathrm{p}$.

\section{RÉSUMÉS}

L'espace franco-germano-suisse du Rhin supérieur dispose d'un maillage de villes performantes: métropole de Mannheim, Eurodistrict de Strasbourg, agglomération trinationale de Bâle... De par sa situation géographique, le sillon rhénan est aussi un couloir de transit d'importance européenne. Une coopération fonctionnelle dans différents domaines du transport comme les aéroports, les gares ou les ports, peut hisser cette région au plan européen et mondial.

Cette étude analyse les interactions entre les projets d'équipements transport et les villes et s'articule autour des points suivants : les métropoles en tant que plates-formes intermodales, les corridors : réalités, projets, enjeux ; les agglomérations et les jeux transfrontaliers. Elle s'efforce de présenter les évolutions d'infrastructures des différents modes de transport en corrélation avec les projets de villes.

The French-German-Swiss area of the upper Rhine offers a meshing of successful cities: metropolis of Mannheim, Eurodistrict of Strasbourg, Basel trinational agglomeration... Because of its geographical situation, the Rhine valley is also a transit corridor of European importance. A functional cooperation in various transport fields such as airports, railway stations or ports, may advance this area to European and worldwide level.

This study analyzes the interactions between cities and transport facilities projects. It is based on the following points: metropolises as intermodal platforms; corridors: realities, projects, stakes; towns and cross-border games, and it tries to present the infrastructure developments of various modes in conjunction with the towns' projects.

\section{INDEX}

Mots-clés : projet urbain, grands équipements de transport, aménagement du territoire, mobilité, intermodalité

Keywords : urban project, major transportation facilities, land use, mobility, intermodality 
AUTEUR

JOËL FORTHOFFER

5, rue Thiergarten, F - 67000 Strasbourg, joel.forthoffer@numericable.fr 